To appear as Ch. 15 in The Oxford Handbook of Spontaneous Thought, K.C.R. Fox and K. Christoff (Editors), forthcoming (2017). New York: Oxford University Press.

\title{
Mind-wandering and self-referential thought
}

\author{
Arnaud D'Argembeau \\ University of Liège, Belgium
}

Address correspondence to: Arnaud D’Argembeau, Department of Psychology, Psychology and Neuroscience of Cognition Research Unit, University of Liège, Quartier Agora, Place des Orateurs 1 (B33), 4000 Liège, Belgium. Email: a.dargembeau@ulg.ac.be

Acknowledgements: Arnaud D'Argembeau is supported by the Fonds de la Recherche Scientifique-FNRS, Belgium. 


\begin{abstract}
When our mind wanders, we frequently experience thoughts, images, and feelings about ourselves and our life. These self-referential thoughts involve diverse contents and take various forms, but most often focus on specific future events that are closely related to our personal goals and concerns. Neuroimaging studies show that such spontaneous mentations recruit many of the same brain regions_-largely corresponding to the default network—as directed self-referential thought. The medial prefrontal cortex is most consistently involved and might contribute to assign value and to integrate processed contents with autobiographical knowledge. The tendency of the wandering mind to focus on self-related information might foster our sense of personal identity and lay the foundation for long-term goal pursuit.
\end{abstract}

Keywords: mind-wandering; task-unrelated thought; spontaneous thought; self; autobiographical memory; goals; future thinking; medial prefrontal cortex; default network 
A fascinating feature of the human mind is its ability to temporarily disengage from current sensory input to mentally simulate alternatives to the here and now. During our daily activities, our minds indeed frequently drift away from the task at hand and focus on various thoughts and mental images that are only loosely (if at all) related to our immediate environment. These thoughts can be remarkably varied in their content and phenomenology, yet a bit of introspection readily indicates that many of them involve self-referential information-memories of personal experiences, anticipations of and plans about one's future, evaluations of one's personal characteristics and life situations, thoughts about one's social relationships, and so on. The purpose of this chapter is to discuss the nature, neural correlates, and possible functions of such self-referential thoughts.

\section{The centrality of self-referential thought when the mind wanders}

The occurrence of thoughts that are not tied to the immediate environment (here referred to as "task-unrelated thought") ${ }^{1}$ is a frequent phenomenon: on average, people's minds wander between 25 and $50 \%$ of the time in daily life, although there is substantial individual and situational variability in this respect (Kane et al., 2007; Killingsworth \& Gilbert, 2010; Song \& Wang, 2012). Furthermore, many of the thoughts (around 30\%) that people experience in their daily life are judged to be spontaneous (Klinger \& Cox, 1987). It has long been proposed that the content of daydreaming and mind-wandering is not random

\footnotetext{
${ }^{1}$ Mind-wandering involves "a shift in the contents of thought away from an ongoing task and/or from events in the external environment to self-generated thoughts and feelings" (Smallwood \& Schooler, 2015). Importantly, however, mind-wandering is a type of thought that is not only characterized by its content but also by its spontaneous and dynamic nature; it involves thoughts that arise freely due to an absence of strong constraints on thought contents and on the transitions from one thought to another (Christoff, Irving, Fox, Spreng, \& AndrewsHanna, 2016). In empirical research on mind-wandering, terms referring to the content (such as "task-unrelated" and "stimulus-independent" thought) or spontaneous nature of mind-wandering are sometimes used interchangeably, which is problematic because these terms designate separable dimensions of thought (Christoff et al., 2016). Whenever possible, in this chapter I use the term "task-unrelated thought" when describing empirical research that investigated mind-wandering from a content-based perspective (i.e., by assessing the contents of thoughts in terms of their relationship to an ongoing task or activity), and the term "spontaneous thought" when describing research that assessed the unconstrained nature of thoughts.
} 
but is often focused on self-relevant information, such as personal goals and concerns (Klinger, 2013; J. L. Singer, 1998; Smallwood \& Schooler, 2006), and recent research on the characteristics of task-unrelated and spontaneous thoughts largely supports this view.

A number of studies have investigated the properties of mind-wandering episodes occurring while performing various tasks in the laboratory. Using a retrospective questionnaire administered after task completion to assess subjective aspects of mindwandering, Andrews-Hanna, Reidler, Huang, and Buckner (2010) found that task-unrelated thoughts were often judged to revolve around personally relevant concerns. Similarly, Stawarczyk, Majerus, Maj, Van der Linden, and D’Argembeau (2011) collected subjective ratings of mind-wandering episodes occurring in the context of a sustained attention task and found that, on average, participants rated their thoughts as personally important and related to their goals. In a study by Baird, Smallwood, and Schooler (2011), participants reported the content of their mental states at several occasions during a choice reaction time task, and the subsequent coding of described thoughts revealed that the majority of task-unrelated thoughts (66\%) included specific mentions of the individual's self. Relatedly, Cole and Berntsen (2016) observed that memories and future thoughts that were spontaneously activated during a vigilance task were frequently linked to the participants' current concerns.

Investigations of how different thought characteristics relate to each other also point to self-relevance as a central dimension of mind-wandering. For example, Stawarczyk, Cassol, and D'Argembeau (2013) had participants rate various characteristics of task-unrelated thoughts and applied a multi-level factor analysis to these data. The results showed that personal significance was an important factor explaining variations in thought characteristics, with dimensions that loaded most strongly on this factor being the importance of thought content, relationship to personal goals, and frequency of occurrence in daily life. In the same vein, other studies have indicated that self-relatedness is a central dimension characterizing 
task-unrelated thought content (Ruby, Smallwood, Engen, \& Singer, 2013; Ruby, Smallwood, Sackur, \& Singer, 2013).

The self-relevance of mind-wandering has likewise been demonstrated in studies investigating the characteristics of thoughts in daily life. Using an experience-sampling method to assess task-unrelated thought content, Song and Wang (2012) found that most mind-wandering episodes were related to one's self and personal life. Andrews-Hanna et al. (2013) asked participants to report and assess a series of thoughts that had been recently in their minds in daily life. Although the characteristics of reported thoughts varied widely, on average, participants characterized their thoughts as highly self-relevant. Furthermore, when examining the relationships between the different characteristics of thoughts using hierarchical clustering analysis, it was found that personal significance was an important dimension characterizing thought content.

Other studies have focused more specifically on particular types of self-related thoughts. For example, in a series of studies by Berntsen and colleagues (e.g., Berntsen, 1996; Berntsen \& Jacobsen, 2008; Finnbogadottir \& Berntsen, 2013), participants monitored the occurrence of involuntary (spontaneous) autobiographical memories and future thoughts in their daily life and recorded their characteristics in a diary. Overall, these studies have shown that involuntary autobiographical memories and future thoughts are common in daily life, are often activated in response to situational cues, and typically arise in states of diffuse attention.

In summary, a number of studies using various methods to assess mind-wandering in the laboratory or in natural settings converge to show that task-unrelated and spontaneous thoughts are frequent and often involve personally significant contents. The next section considers more deeply the different forms that these self-related thoughts can take. 


\section{Varieties of self-referential thought}

Self-referential thoughts can vary widely in their content, specificity, and temporal orientation. Some thoughts rely on personal semantic information, such as abstract representations of one's personal characteristics (e.g., one's personality traits, abilities, goals, social roles, preferences, and values) and knowledge of facts about one's life (Klein \& Gangi, 2010; Markus \& Wurf, 1987; Renoult, Davidson, Palombo, Moscovitch, \& Levine, 2012). Other thoughts involve the mental simulation of specific life episodes, either events that occurred in one's personal past or situations that might happen in one's personal future (D’Argembeau \& Van der Linden, 2004; Schacter, 2012; Szpunar, 2010). Although the extent to which the wandering mind involves these different types of self-referential thought remains to be investigated in detail, current evidence suggests that it tends preferentially toward particular forms.

One of the most consistent findings has been that the wandering mind focuses most frequently on future events. For example, Smallwood, Nind, and O'Connor (2009) observed that task-unrelated thoughts that occurred while performing an undemanding task were mostly future-oriented, a finding that has been replicated in several laboratory studies of mindwandering (Baird et al., 2011; Ruby, Smallwood, Engen, et al., 2013; Ruby, Smallwood, Sackur, et al., 2013; Smallwood et al., 2011; Stawarczyk et al., 2013; Stawarczyk, Majerus, Maj, et al., 2011). Similarly, studies investigating the characteristics of task-unrelated thoughts in daily life have shown that most thoughts focus on the future (Andrews-Hanna et al., 2013; Song \& Wang, 2012). Furthermore, there is evidence that future-oriented mindwanderings are perceived as more personally significant than non-future-oriented mindwanderings (Stawarczyk et al., 2013), and often involve the anticipation and planning of personally relevant future goals (Baird et al., 2011). 
The future-orientation of task-unrelated thoughts seems particularly pronounced when self-relevant information is salient in a person's mind. Indeed, research has shown that priming personally significant contents increases the prospective bias of mind-wandering. Stawarczyk, Majerus, Maj et al. (2011) asked participants to write an essay about their personal goals or about a topic unrelated to personal goals (i.e., a familiar itinerary); then, the temporal focus of mind-wandering during an unrelated task was assessed. The results showed that participants who had previously thought about their personal goals subsequently experienced more future-oriented mind-wandering. In a related vein, Smallwood et al. (2011) found that making people think about their personality traits increased future-oriented mindwandering in a subsequent task.

Other data suggest that, although task-unrelated thoughts can vary widely in their representational format and degree of abstraction, they most often focus on specific events. Andrews-Hanna et al. (2013) found that $77 \%$ of thoughts reported to happen in daily life pertained to a specific event and, of these, $60 \%$ were oriented towards the future. Similarly, Stawarczyk et al. (2013) found that the contents of mind-wandering episodes were mostly related to something concrete and well-defined (e.g., a particular situation or action). Some studies have further compared the characteristics of spontaneous versus voluntary autobiographical representations, showing that spontaneous memories and future thoughts are typically more specific than their voluntary counterparts (Berntsen \& Jacobsen, 2008; Cole, Staugaard, \& Berntsen, 2015).

Altogether, these findings indicate that the wandering mind can represent diverse contents and take various forms, but most often involves the representation of specific future events that are closely related to one's personal goals (for a more in-depth discussion, see the chapter by Stawarczyk in this volume). 


\section{Neural correlates of directed and spontaneous self-referential thought}

Neuroimaging research has shown that mind-wandering is associated with activations in a specific set of brain regions, which largely corresponds to the so-called default network (Buckner, Andrews-Hanna, \& Schacter, 2008) and includes the medial prefrontal cortex, posterior cingulate/precuneus, inferior parietal lobules, and areas of the medial and lateral temporal lobes (e.g., Christoff, Gordon, Smallwood, Smith, \& Schooler, 2009; Mason et al., 2007; Stawarczyk, Majerus, Maquet, \& D’Argembeau, 2011) (for meta-analyses, see Fox, Spreng, Ellamil, Andrews-Hanna, \& Christoff, 2015; Stawarczyk \& D’Argembeau, 2015). Many of these regions are also activated when people are explicitly instructed to process selfrelated information, which provides additional support to the view that self-referential thought is an important component of the mind-wandering state. This section first presents an overview of the neural correlates of different types of self-representation (as revealed by taskbased studies), and then considers the few studies that have investigated the neural correlates of task-unrelated and spontaneous self-referential thought.

The neural correlates of different types of self-representation have been extensively investigated using functional neuroimaging. Some studies have focused on personal semantic knowledge, such as the representation of one's personality traits. In a typical study (e.g., Kelley et al., 2002), participants are asked to evaluate the self-descriptiveness of traits (e.g., polite, dependable, daring) and this task is compared to making other types of trait judgements (e.g., assessing their descriptiveness in reference to another person). Several meta-analyses have shown that both self- and other-referential judgments engage regions supporting semantic processing (such as the lateral temporal cortex), and the medial prefrontal cortex (mPFC) is typically more activated during self- vs. other-referential judgments 
(Araujo, Kaplan, \& Damasio, 2013; Murray, Schaer, \& Debbane, 2012; van der Meer, Costafreda, Aleman, \& David, 2010). Research further suggests that the mPFC is involved in processing different types of personal semantic information: not only one's personality traits, but also one's attitudes, values, and physical attributes (e.g., Brosch, Coppin, Schwartz, \& Sander, 2012; Jenkins \& Mitchell, 2011; Zysset, Huber, Ferstl, \& von Cramon, 2002).

The neural basis of autobiographical memory—memories of one's past experiences and knowledge of facts about one's life — has also received extensive attention (for a review, see Cabeza \& St Jacques, 2007). In many studies, memories of specific personal experiences (i.e., events that happened at a particular place and time in an individual's life) are compared with the retrieval of non-personal information (e.g., non-personal semantic knowledge or stimuli that have been learned in the laboratory before the scanning session). Neuroimaging evidence indicates that such autobiographical memory retrieval is associated with activations in the $\mathrm{mPFC}$, medial and lateral temporal areas, posterior cingulate/retrosplenial cortex, and inferior parietal lobes (for meta-analyses, see Kim, 2012; Martinelli, Sperduti, \& Piolino, 2013; McDermott, Szpunar, \& Christ, 2009; Spreng, Mar, \& Kim, 2009; Svoboda, McKinnon, \& Levine, 2006). Furthermore, lesion data have revealed that damage to these areas is associated with deficits in autobiographical memory retrieval (Philippi, Tranel, Duff, \& Rudrauf, 2015).

A recent meta-analysis of neuroimaging studies (Martinelli et al., 2013) has compared the neural correlates of different types of self-referential thought-representations of personal characteristics (such as personality traits), knowledge of personal facts, and memories for specific events. The results show that each type of self-referential thought is associated with unique brain activations, with a shift from posterior to anterior regions with increasing abstraction of representations. Indeed, specific memories predominantly activate posterior structures, including the medial and lateral temporal lobes and posterior cingulate/precuneus, 
whereas abstract representations of personal characteristics only recruit medial prefrontal structures; finally, knowledge of personal facts is associated with anterior activations, as well as posterior structures (mainly the lateral temporal cortex). Interestingly, the mPFC is the only brain region that is consistently activated when thinking about one's traits, retrieving specific experiences from one's past, and accessing knowledge of facts about one's life, with both common and distinct activations across these three kinds of self-referential thought.

As mentioned in the previous section, the wandering mind often focuses on future events and involves the processing of personal goals. The neural correlates of future-oriented and goal-directed thought are thus of particular interest. Neuroimaging studies in which participants are explicitly instructed to reflect on their personal goals have shown that this process recruits areas of the default network. For example, Johnson et al. (2006) asked participants to think about their hopes and aspirations, about their duties and obligations, and about non-self-relevant topics (e.g., polar bear fishing). The results showed that the mPFC, posterior cingulate/precuneus, and lateral temporal cortex were more activated when thinking about personal goals than when thinking about non-self-relevant topics. There were also distinct activations as a function of the type of personal goals considered: a region of the mPFC showed greater activation when thinking about hopes and aspirations, whereas a posterior medial region showed greater activation when thinking about duties and obligations. Subsequent studies have detected similar activations in the $\mathrm{mPFC}$, posterior cingulate, and lateral temporal cortex when reflecting on personal goals, especially promotion goals (i.e., things the individual would like to achieve) (for a meta-analysis, see Stawarczyk \& D’Argembeau, 2015).

In recent years there has also been increasing interest in episodic future thinking - the ability to imagine or simulate specific events that might happen in one's personal future 
(D’Argembeau, 2012; Schacter, Addis, \& Buckner, 2008; Szpunar, 2010). A number of neuroimaging studies have shown that such mental simulations of future events depend on largely the same brain regions as autobiographical remembering - the mPFC, posterior cingulate and restrosplenial cortices, medial and lateral temporal regions, inferior parietal lobules, and parts of the lateral prefrontal cortex (e.g., Addis, Wong, \& Schacter, 2007; Szpunar, Watson, \& McDermott, 2007; for a meta-analysis, see Benoit \& Schacter, 2015). These different brain regions likely support different component processes involved in episodic future thought; for example, the medial temporal lobe may support the flexible retrieval and recombination of episodic details to construct representations of novel events (Addis \& Schacter, 2012), whereas the lateral temporal cortex may represent semantic knowledge that is used for constructing coherent events (Irish, Addis, Hodges, \& Piguet, 2012).

A few studies have investigated whether personal goal processing is associated with specific activations within this core network supporting episodic future thought. It has been found that medial prefrontal and posterior cingulate cortices are more activated when thinking about goal-related compared to goal-unrelated future events, suggesting that cortical midline structures may underpin the processing of personal goals during episodic future thinking (D’Argembeau et al., 2010). In line with this view, a recent meta-analysis has shown that studies of episodic future thinking and studies of personal goal processing are associated with overlapping activations in the mPFC and posterior cingulate cortex, as well as other regions of the core network (Stawarczyk \& D'Argembeau, 2015). It has also been found that imagining taking various steps and actions to achieve personal goals depends on the functional coupling of the core network with the frontoparietal control network, a system supporting cognitive control (Spreng, Stevens, Chamberlain, Gilmore, \& Schacter, 2010). Thus, the frontoparietal control network may also contribute to personal goal processing, perhaps by monitoring and 
integrating future-oriented thoughts in coherent sequences to achieve imagined end-states. A recent study has further clarified the role of distinct brain areas in personal goal processing by showing that imagining what it would be like to achieve a given goal mainly involves the mPFC, whereas constructing a detailed plan of how to reach this goal engages regions from both the core network and the frontroparietal control network (Gerlach, Spreng, Madore, \& Schacter, 2014).

While many studies have investigated the neural correlates of self-referential thought using directed self-processing tasks, only a few studies have examined the brain regions associated with task-unrelated and spontaneous self-referential thought. Some studies investigated thought processes spontaneously occurring when people were not required to perform a particular task (the so-called resting state). In a pioneering study, Andreasen et al. (1995) used positron emission tomography (PET) to investigate similarities and differences in neural activity between the explicit retrieval of autobiographical memories and a rest condition (i.e., lying quietly with no specific instructions about mental activity). They found that the mPFC and medial posterior areas (precuneus/retrosplenial cortex) showed greater activity during both autobiographical memory retrieval and rest compared to a semantic memory condition. Interviews with the participants indicated that they spontaneously thought about a variety of things during the rest condition, but especially about self-related contents such as past experiences and future activities. The authors concluded that the psychological commonality between the rest and autobiographical memory conditions is that "both involve something personal and highly individual” (p. 1583).

Another PET study examined the commonalities in brain activation between the resting state and the active reflection on one's personality traits (D'Argembeau et al., 2005). It was found that both conditions were associated with common activation in the mPFC compared with conditions requiring participants to reflect on non-self-related contents. 
Furthermore, an analysis of the content of mental activity (using verbal reports and rating scales obtained after each scan) showed that participants spontaneously experienced selfreferential thought during the rest condition, and the amount of self-referential processing correlated specifically with the activity of the mPFC. Common activations in the mPFC and posterior cingulate cortex during the resting state and directed self-reference tasks have also been observed in a more recent fMRI study (Whitfield-Gabrieli et al., 2011), and have been confirmed by a meta-analysis comparing the location of activations in neuroimaging studies on the default network (i.e., brain regions showing stronger activation during the resting state compared to active tasks) with the location of activations associated with various self-related tasks (e.g., trait judgments, autobiographical memory, face recognition, and name perception) (Qin \& Northoff, 2011).

There is also evidence that self-referential thoughts that occur while performing an unrelated task are associated with similar neural correlates as directed self-referential thoughts. Stawarczyk and D’Argembeau (2015) performed a meta-analysis of neuroimaging studies of episodic future thinking on the one hand, and a meta-analysis of neuroimaging studies of mind-wandering on the other hand. A conjunction analysis showed that these two types of studies were associated with common activations in several regions of the default network, including the mPFC, posterior cingulate cortex, left inferior parietal cortex, and left medial and lateral temporal regions. Considering that most mind-wandering episodes are future-oriented (see above), this finding suggests that task-based and task-unrelated future thoughts are supported by largely similar brain regions. Some differences between the two kinds of self-referential thoughts were also noted, however. In particular, the lateral prefrontal cortex showed greater activity during directed future thinking compared to mind-wandering. Similarly, it has been found that voluntary episodic memories involve the lateral prefrontal cortex to a greater extent than involuntary episodic memories, with otherwise extensive 
overlaps in default network activity between the two kinds of memories (Hall et al., 2014). Together, these results suggest that directed and spontaneous self-referential thoughts share many commonalities, but may differ in the extent to which they rely on effortful cognitive processes (see also Dixon, Fox, \& Christoff, 2014).

In summary, neuroimaging studies have shown that directed self-referential thoughts are associated with activations in a set of brain regions that largely corresponds to the default network. The specific areas that are recruited depend on the type of self-representation under consideration, with abstract thoughts relying mainly on the mPFC and lateral temporal cortex, while more specific (past or future) thoughts recruit additional regions supporting the representation of episodic details (e.g., the medial temporal lobe and posterior cingulate/restroplenial cortex). A number of studies and meta-analyses have further demonstrated that many of the same areas are activated during the resting state or in association with mind-wandering, suggesting that these brain regions are involved in spontaneous or unconstrained self-referential thought processes. Across all these studies, the $\mathrm{mPFC}$ is the brain region that has been most consistently associated with different types of self-representation, indicating that this region might play a particularly important role in both intentional and spontaneous self-referential cognition.

\section{What is the role of the medial prefrontal cortex in self-referential thought?}

While it has become clear that the mPFC plays a key role in self-referential thought, the exact processing operations mediated by this region are not fully understood. One possibility is that the mPFC contributes to appraise or represent the subjective value or significance of self-related information. Indeed, the mPFC is thought to play a broad role in affective and value-based processing (Roy, Shohamy, \& Wager, 2012). Most notably, 
research has shown that medial prefrontal activity encodes the subjective values of various types of rewards, which has led to the view that the $\mathrm{mPFC}$ integrates information from multiple sources to represent the significance or value of stimuli (for review, see Levy \& Glimcher, 2012; Peters \& Buchel, 2010b; Rangel \& Hare, 2010; Sescousse, Caldu, Segura, \& Dreher, 2013). Although these studies focused on the subjective valuation of stimuli from the external environment, the medial prefrontal activations that are observed in relation to selfreferential thought could reflect a similar valuation mechanism (D'Argembeau, 2013). Stimuli and mental representations that refer or relate to the self tend to be assigned unique value, and the function of the mPFC may precisely be to evaluate or represent such significance. This account is, for example, supported by the finding that activity in the mPFC increases linearly with the personal importance of the mental representations under consideration (AndrewsHanna, Reidler, Sepulcre, Poulin, \& Buckner, 2010; D’Argembeau et al., 2012). By flexibly assigning degrees of value to self-related contents, the mPFC might play an important role in the construction, stabilization, and modification of self-representations.

Another (not necessarily mutually exclusive but somewhat broader) view is that the mPFC supports the integration of multiple representations during self-referential thought. It has been proposed that self-reference acts as an integrative hub for perception, decision making, and memory, helping to bind together different types of information and even different stages of processing (Sui \& Humphreys, 2015). In support of this view, there is for example evidence that people recall more episodic details about items judged in reference to the self, suggesting that self-reference enhances the binding of different forms of information in memory (e.g., items and associated contextual details) (Conway, Dewhurst, Pearson, \& Sapute, 2001). A potential role of the mPFC in this integrative process is suggested by evidence showing that the self-reference effect in memory is predicted by medial prefrontal activity in healthy individuals (Macrae, Moran, Heatherton, Banfield, \& Kelley, 2004) and is 
abolished in patients with focal brain damage to the mPFC (Philippi, Duff, Denburg, Tranel, \& Rudrauf, 2012). More generally, there is substantial evidence that the mPFC is involved in relating and integrating incoming information to pre-existing knowledge structures (Brod, Werkle-Bergner, \& Shing, 2013; Kroes \& Fernández, 2012; Preston \& Eichenbaum, 2013; van Kesteren, Ruiter, Fernández, \& Henson, 2012).

The integration of multiple representations may be particularly important for autobiographical remembering and future thinking, which inherently involve different types or levels of self-related information (Conway, 2005; D’Argembeau \& Mathy, 2011). For example, there is evidence that specific memories and future thoughts are often part of higherorder autobiographical knowledge structures that organize specific events in broader themes and causal sequences — referred to as event clusters (Brown \& Schopflocher, 1998;

D’Argembeau \& Demblon, 2012). In a recent fMRI study, we found that the processing of such event clusters is associated with increased activation in the $\mathrm{MPFC}$ and with greater functional coupling between the mPFC and posterior regions supporting semantic and episodic representations (Demblon, Bahri, \& D’Argembeau, 2016). These findings suggest that the function of the mPFC during autobiographical remembering and future thinking might be to integrate specific event representations with higher-order autobiographical knowledge (e.g., personal goals and general knowledge about one's life). Through this integrative process, the mPFC might contribute to contextualize specific event representations within an individual's life story, thus rendering memories and future thoughts truly autobiographical.

\section{On the possible functions of spontaneous self-referential thought}


The fact that mind-wandering is such a pervasive experience makes it unlikely that it merely consists in a lapse of attention with no intrinsic value. On the contrary, there is growing evidence suggesting that mind-wandering may serve adaptive functions, such as planning (Baird et al., 2011) and problem solving (Ruby, Smallwood, Sackur, et al., 2013).

The main function of mind-wandering might be to allow the mental simulation of alternatives to the here and now, and in particular to envision possible futures. As mentioned above, most mind-wandering episodes indeed involve future-oriented and goal-related contents. The wandering mind may anticipate a variety of future events, conceive possible ways to attain or avoid envisioned situations, and predict the probable outcomes of different courses of action. In turn, these mental simulations may be used to inform decisions and behaviors. Many mind-wandering episodes are subjectively perceived as fulfilling such future-oriented functions - evaluating and planning for possible situations, making decisions, and solving problems (Stawarczyk et al., 2013; Stawarczyk, Majerus, Maj, et al., 2011). Although the extent to which such future-oriented thoughts are beneficial in guiding decisions and behavior remains to be investigated in detail, recent evidence suggests that this might be the case. For example, a recent study suggests that mind-wandering can help people to better specify their goals and plans, which might lead to more effective goal achievement (Medea et al., 2016). Furthermore, it has also been found that mind-wandering (Smallwood, Ruby, \& Singer, 2013) and future thinking (Lin \& Epstein, 2014) are associated with reduced delay discounting (i.e., with a greater capacity to resist the temptation of an immediate reward in favor of receiving a larger reward later in the future). Interestingly, neuroimaging studies suggest that the mPFC might mediate this effect of future-oriented thoughts on farsighted decisions (Benoit, Gilbert, \& Burgess, 2011; Bernhardt et al., 2014; Peters \& Buchel, 2010a).

Another potentially important function of mind-wandering may be to contribute to our sense of personal identity. People in modern societies construct their personal identity by 
creating an evolving life story that integrates past, present, and possible future experiences in such a way as to provide their lives with some degree of unity, purpose, and meaning (McAdams, 2001; J. A. Singer, Blagov, Berry, \& Oost, 2013). The construction of such narratives critically depends on autobiographical reasoning, a process of reflective thinking through which we form links between disparate elements of our life and the self (Habermas \& Bluck, 2000). Autobiographical reasoning helps in establishing personal identity and continuity across change, and research has shown the importance of this process for identity development, maturity, and well-being (King, Scollon, Ramsey, \& Williams, 2000;

Lilgendahl \& McAdams, 2011; McLean \& Pratt, 2006; Raffard et al., 2010; J. A. Singer, Rexhaj, \& Baddeley, 2007). Intriguingly, a recent study (D’Argembeau et al., 2014) has shown that the neural correlates of autobiographical reasoning closely correspond to a dorsomedial prefrontal subsystem of the default network, which might play a broad role in introspective processes (Andrews-Hanna, 2012). Although this issue remains to be investigated in detail, this might suggest that spontaneous self-referential thought plays a role in reflecting on the broader meaning and implications of personal experiences, thereby contributing to the construction, maintenance, and update of an individual's life story. In addition, frequent mental trips to the past and future during the mind-wandering state might also provide a more direct, experiential sense of continuity of the self through time (Prebble, Addis, \& Tippett, 2013). Through these processes, the wandering mind might thus contribute to the creation and functional maintenance of self-models, which then lay the foundation for long-term motivation and future planning (Metzinger, 2013).

\section{Conclusion}


When our attention is not entirely focused on the external environment, various thoughts and mental images tend to spontaneously populate our minds. These spontaneous mentations frequently involve personally significant contents and often focus on future, goalrelated events. Neuroimaging research suggests that the activity of a specific set of brain regions, largely corresponding to the default network, correlates with the formation of selfreferential thoughts during the mind-wandering or resting state. The specific areas that are recruited depend on the content and specificity of the mental representation under consideration, but the mPFC appears to play a broad role in processing various types of selfrelated contents. While the specific function of this brain region remains to be investigated in detail, current evidence suggests that it might process the subjective value of self-related information and/or integrate multiple representations in the service of self-referential thought. Overall, the tendency of the wandering mind to focus on self-related contents may foster one's sense of personal identity and serve adaptive functions, such as planning and long-term goal pursuit. 


\section{References}

Addis, D. R., \& Schacter, D. L. (2012). The hippocampus and imagining the future: where do we stand? Front Hum Neurosci, 5, 173.

Addis, D. R., Wong, A. T., \& Schacter, D. L. (2007). Remembering the past and imagining the future: Common and distinct neural substrates during event construction and elaboration. Neuropsychologia, 45, 1363-1377.

Andreasen, N. C., O'Leary, D. S., Cizadlo, T., Arndt, S., Rezai, K., Watkins, G. L., ... Hichwa, R. D. (1995). Remembering the past: two facets of episodic memory explored with positron emission tomography. Am J Psychiatry, 152(11), 1576-85.

Andrews-Hanna, J. R. (2012). The brain's default network and its adaptive role in internal mentation. Neuroscientist, 18(3), 251-70.

Andrews-Hanna, J. R., Kaiser, R. H., Turner, A. E. J., Reineberg, A., Godinez, D., Dimidjian, S., \& Banich, M. (2013). A penny for your thoughts: dimensions of self-generated thought content and relationships with individual differences in emotional wellbeing. Front Psychol, 4, 900.

Andrews-Hanna, J. R., Reidler, J. S., Huang, C., \& Buckner, R. L. (2010). Evidence for the default network's role in spontaneous cognition. J Neurophysiol, 104(1), 322-35.

Andrews-Hanna, J. R., Reidler, J. S., Sepulcre, J., Poulin, R., \& Buckner, R. L. (2010). Functionalanatomic fractionation of the brain's default network. Neuron, 65(4), 550-62.

Araujo, H. F., Kaplan, J., \& Damasio, A. (2013). Cortical midline structures and autobiographical-self processes: an activation-likelihood estimation meta-analysis. Frontiers in Human Neuroscience, 7, 548.

Baird, B., Smallwood, J., \& Schooler, J. W. (2011). Back to the future: autobiographical planning and the functionality of mind-wandering. Consciousness and Cognition, 20(4), 1604-1611.

Benoit, R. G., Gilbert, S. J., \& Burgess, P. W. (2011). A neural mechanism mediating the impact of episodic prospection on farsighted decisions. The Journal of Neuroscience, 31(18), 67716779. 
Benoit, R. G., \& Schacter, D. L. (2015). Specifying the core network supporting episodic simulation and episodic memory by activation likelihood estimation. Neuropsychologia, 75, 450-457.

Bernhardt, B. C., Smallwood, J., Tusche, A., Ruby, F. J. M., Engen, H. G., Steinbeis, N., \& Singer, T. (2014). Medial prefrontal and anterior cingulate cortical thickness predicts shared individual differences in self-generated thought and temporal discounting. Neurolmage, 90, 290-297.

Berntsen, D. (1996). Involuntary autobiographical memories. Applied Cognitive Psychology, 10, 435454.

Berntsen, D., \& Jacobsen, A. S. (2008). Involuntary (spontaneous) mental time travel into the past and future. Conscious.Cogn, 17, 1093-1104.

Brod, G., Werkle-Bergner, M., \& Shing, Y. L. (2013). The influence of prior knowledge on memory: a developmental cognitive neuroscience perspective. Frontiers in Behavioral Neuroscience, 7, 139.

Brosch, T., Coppin, G., Schwartz, S., \& Sander, D. (2012). The importance of actions and the worth of an object: dissociable neural systems representing core value and economic value. Soc Cogn Affect Neurosci, 7(5), 497-505.

Brown, N. R., \& Schopflocher, D. (1998). Event clusters: An organization of personal events in autobiographical memory. Psychological Science, 9(6), 470-475.

Buckner, R. L., Andrews-Hanna, J. R., \& Schacter, D. L. (2008). The brain's default network - Anatomy, function, and relevance to disease. Ann N Y Acad Sci., 1124, 1-38.

Cabeza, R., \& St Jacques, P. (2007). Functional neuroimaging of autobiographical memory. Trends Cogn Sci., 11(5), 219-227.

Christoff, K., Gordon, A. M., Smallwood, J., Smith, R., \& Schooler, J. W. (2009). Experience sampling during fMRI reveals default network and executive system contributions to mind wandering. Proc Natl Acad Sci U S A, 106(21), 8719-24. 
Christoff, K., Irving, Z. C., Fox, K. C. R., Spreng, R. N., \& Andrews-Hanna, J. R. (2016). Mind-wandering as spontaneous thought: a dynamic framework. Nature Reviews Neuroscience, advance online publication. https://doi.org/10.1038/nrn.2016.113

Cole, S. N., \& Berntsen, D. (2016). Do future thoughts reflect personal goals? Current concerns and mental time travel into the past and future. The Quarterly Journal of Experimental Psychology, 69(2), 273-284.

Cole, S. N., Staugaard, S. R., \& Berntsen, D. (2015). Inducing involuntary and voluntary mental time travel using a laboratory paradigm. Memory \& Cognition.

Conway, M. A. (2005). Memory and the self. Journal of Memory and Language, 53, 594-628.

Conway, M. A., Dewhurst, S. A., Pearson, N., \& Sapute, A. (2001). The self and recollection reconsidered: how a "failure to replicate" failed and why trace strength accounts of recollection are untenable. Applied Cognitive Psychology, 15(6), 673-686.

D’Argembeau, A. (2012). Autobiographical memory and future thinking. In D. Berntsen \& D. C. Rubin (Eds.), Understanding autobiographical memory: Theories and approaches (pp. 311-330). Cambridge University Press.

D'Argembeau, A. (2013). On the role of the ventromedial prefrontal cortex in self-processing: the valuation hypothesis. Frontiers in Human Neuroscience, 7, 372.

D’Argembeau, A., Cassol, H., Phillips, C., Balteau, E., Salmon, E., \& Van der Linden, M. (2014). Brains creating stories of selves: the neural basis of autobiographical reasoning. Soc Cogn Affect Neurosci, 9, 646-652.

D’Argembeau, A., Collette, F., Van der Linden, M., Laureys, S., Del Fiore, G., Degueldre, C., ... Salmon, E. (2005). Self-referential reflective activity and its relationship with rest: a PET study. Neuroimage, 25(2), 616-624.

D’Argembeau, A., \& Demblon, J. (2012). On the representational systems underlying prospection: Evidence from the event-cueing paradigm. Cognition, 125(2), 160-167. 
D’Argembeau, A., Jedidi, H., Balteau, E., Bahri, M., Phillips, C., \& Salmon, E. (2012). Valuing one's self: medial prefrontal involvement in epistemic and emotive investments in self-views. Cereb Cortex, 22(3), 659-67.

D'Argembeau, A., \& Mathy, A. (2011). Tracking the construction of episodic future thoughts. Journal of Experimental Psychology: General, 140, 258-271.

D’Argembeau, A., Stawarczyk, D., Majerus, S., Collette, F., Van der Linden, M., Feyers, D., ... Salmon, E. (2010). The neural basis of personal goal processing when envisioning future events. J Cogn Neurosci, 22(8), 1701-13.

D'Argembeau, A., \& Van der Linden, M. (2004). Phenomenal characteristics associated with projecting oneself back into the past and forward into the future: influence of valence and temporal distance. Conscious. Cogn, 13(4), 844-858.

Demblon, J., Bahri, M. A., \& D’Argembeau, A. (2016). Neural correlates of event clusters in past and future thoughts: How the brain integrates specific episodes with autobiographical knowledge. Neurolmage, 127, 257-266.

Dixon, M. L., Fox, K. C. R., \& Christoff, K. (2014). A framework for understanding the relationship between externally and internally directed cognition. Neuropsychologia, 62, 321-330.

Finnbogadottir, H., \& Berntsen, D. (2013). Involuntary future projections are as frequent as involuntary memories, but more positive. Conscious Cogn, 22(1), 272-80.

Fox, K. C. R., Spreng, R. N., Ellamil, M., Andrews-Hanna, J. R., \& Christoff, K. (2015). The wandering brain: Meta-analysis of functional neuroimaging studies of mind-wandering and related spontaneous thought processes. Neurolmage, 111, 611-621.

Gerlach, K. D., Spreng, R. N., Madore, K. P., \& Schacter, D. L. (2014). Future planning: default network activity couples with frontoparietal control network and reward-processing regions during process and outcome simulations. Soc Cogn Affect Neurosci, 9(12), 1942-51.

Habermas, T., \& Bluck, S. (2000). Getting a life: the emergence of the life story in adolescence. Psychol Bull, 126(5), 748-69. 
Hall, S. A., Rubin, D. C., Miles, A., Davis, S. W., Wing, E. A., Cabeza, R., \& Berntsen, D. (2014). The neural basis of involuntary episodic memories. Journal of Cognitive Neuroscience, 26(10), 2385-2399.

Irish, M., Addis, D. R., Hodges, J. R., \& Piguet, O. (2012). Considering the role of semantic memory in episodic future thinking: evidence from semantic dementia. Brain, 135(Pt 7), 2178-91.

Jenkins, A. C., \& Mitchell, J. P. (2011). Medial prefrontal cortex subserves diverse forms of selfreflection. Soc Neurosci, 6(3), 211-8.

Johnson, M. K., Raye, C. L., Mitchell, K. J., Touryan, S. R., Greene, E. J., \& Nolen-Hoeksema, S. (2006). Dissociating medial frontal and posterior cingulate activity during self-reflection. Soc Cogn Affect Neurosci, 1(1), 56-64.

Kane, M. J., Brown, L. H., McVay, J. C., Silvia, P. J., Myin-Germeys, I., \& Kwapil, T. R. (2007). For whom the mind wanders, and when - An experience-sampling study of working memory and executive control in daily life. Psychological Science, 18(7), 614-621.

Kelley, W. M., Macrae, C. N., Wyland, C. L., Caglar, S., Inati, S., \& Heatherton, T. F. (2002). Finding the self? An event-related fMRI study. J Cogn Neurosci, 14, 785-794.

Killingsworth, M. A., \& Gilbert, D. T. (2010). A wandering mind is an unhappy mind. Science, 330(6006), 932.

Kim, H. (2012). A dual-subsystem model of the brain's default network: Self-referential processing, memory retrieval processes, and autobiographical memory retrieval. Neuroimage, 61(4), 966-77.

King, L. A., Scollon, C. K., Ramsey, C., \& Williams, T. (2000). Stories of life transition: Subjective wellbeing and ego development in parents of children with Down Syndrome. Journal of Research in Personality, 34, 509-536.

Klein, S. B., \& Gangi, C. E. (2010). The multiplicity of self: neuropsychological evidence and its implications for the self as a construct in psychological research. Ann N Y Acad Sci, 1191, 115. 
Klinger, E. (2013). Goal Commitments and the content of thoughts and dreams: basic principles. Front Psychol, 4, 415.

Klinger, E., \& Cox, W. M. (1987). Dimensions of thought flow in everyday life. Imagination, Cognition, and Personality, 7, 105-128.

Kroes, M. C. W., \& Fernández, G. (2012). Dynamic neural systems enable adaptive, flexible memories. Neuroscience and Biobehavioral Reviews, 36(7), 1646-1666.

Levy, D. J., \& Glimcher, P. W. (2012). The root of all value: a neural common currency for choice. Curr Opin Neurobiol, 22(6), 1027-38.

Lilgendahl, J. P., \& McAdams, D. P. (2011). Constructing stories of self-growth: How individual differences in patterns of autobiographical reasoning relate to well-being in midlife. Journal of Personality, 79, 391-428.

Lin, H., \& Epstein, L. H. (2014). Living in the moment: effects of time perspective and emotional valence of episodic thinking on delay discounting. Behavioral Neuroscience, 128(1), 12-19.

Macrae, C. N., Moran, J. M., Heatherton, T. F., Banfield, J. F., \& Kelley, W. M. (2004). Medial prefrontal activity predicts memory for self. Cereb.Cortex, 14(6), 647-654.

Markus, H., \& Wurf, E. (1987). The dynamic self-concept: A social psychological perspective. Annual Review of Psychology, 38, 299-337.

Martinelli, P., Sperduti, M., \& Piolino, P. (2013). Neural substrates of the self-memory system: new insights from a meta-analysis. Hum Brain Mapp, 34(7), 1515-29.

Mason, M. F., Norton, M. I., Van Horn, J. D., Wegner, D. M., Grafton, S. T., \& Macrae, C. N. (2007). Wandering minds: the default network and stimulus-independent thought. Science, 315(5810), 393-395.

McAdams, D. P. (2001). The psychology of life stories. Review of General Psychology, 5, 100-122.

McDermott, K. B., Szpunar, K. K., \& Christ, S. E. (2009). Laboratory-based and autobiographical retrieval tasks differ substantially in their neural substrates. Neuropsychologia, 47(11), 22908. 
McLean, K. C., \& Pratt, M. W. (2006). Life's little (and big) lessons: identity statuses and meaningmaking in the turning point narratives of emerging adults. Dev Psychol, 42(4), 714-22.

Medea, B., Karapanagiotidis, T., Konishi, M., Ottaviani, C., Margulies, D., Bernasconi, A., ... Smallwood, J. (2016). How do we decide what to do? Resting-state connectivity patterns and components of self-generated thought linked to the development of more concrete personal goals. Experimental Brain Research, 1-13.

Metzinger, T. (2013). The myth of cognitive agency: subpersonal thinking as a cyclically recurring loss of mental autonomy. Frontiers in Psychology, 4, 931.

Murray, R. J., Schaer, M., \& Debbane, M. (2012). Degrees of separation: a quantitative neuroimaging meta-analysis investigating self-specificity and shared neural activation between self- and other-reflection. Neurosci Biobehav Rev, 36(3), 1043-59.

Peters, J., \& Buchel, C. (2010a). Episodic future thinking reduces reward delay discounting through an enhancement of prefrontal-mediotemporal interactions. Neuron, 66(1), 138-48.

Peters, J., \& Buchel, C. (2010b). Neural representations of subjective reward value. Behav Brain Res, 213(2), 135-41.

Philippi, C. L., Duff, M. C., Denburg, N. L., Tranel, D., \& Rudrauf, D. (2012). Medial PFC damage abolishes the self-reference effect. J Cogn Neurosci, 24(2), 475-81.

Philippi, C. L., Tranel, D., Duff, M., \& Rudrauf, D. (2015). Damage to the default mode network disrupts autobiographical memory retrieval. Social Cognitive and Affective Neuroscience, 10(3), 318-326.

Prebble, S. C., Addis, D. R., \& Tippett, L. J. (2013). Autobiographical Memory and Sense of Self. Psychol Bull, 139, 815-840.

Preston, A. R., \& Eichenbaum, H. (2013). Interplay of Hippocampus and Prefrontal Cortex in Memory. Current Biology, 23(17), R764-R773.

Qin, P., \& Northoff, G. (2011). How is our self related to midline regions and the default-mode network? Neuroimage, 57(3), 1221-33. 
Raffard, S., D’Argembeau, A., Lardi, C., Bayard, S., Boulenger, J. P., \& Van der Linden, M. (2010). Narrative identity in schizophrenia. Conscious Cogn, 19(1), 328-40.

Rangel, A., \& Hare, T. (2010). Neural computations associated with goal-directed choice. Curr Opin Neurobiol, 20(2), 262-70.

Renoult, L., Davidson, P. S., Palombo, D. J., Moscovitch, M., \& Levine, B. (2012). Personal semantics: at the crossroads of semantic and episodic memory. Trends Cogn Sci, 16(11), 550-8.

Roy, M., Shohamy, D., \& Wager, T. D. (2012). Ventromedial prefrontal-subcortical systems and the generation of affective meaning. Trends Cogn Sci, 16(3), 147-56.

Ruby, F. J. M., Smallwood, J., Engen, H., \& Singer, T. (2013). How Self-Generated Thought Shapes Mood-The Relation between Mind-Wandering and Mood Depends on the Socio-Temporal Content of Thoughts. PLOS ONE, 8(10), e77554.

Ruby, F. J. M., Smallwood, J., Sackur, J., \& Singer, T. (2013). Is self-generated thought a means of social problem solving? Front Psychol, 4, 962.

Schacter, D. L. (2012). Adaptive constructive processes and the future of memory. Am Psychol, 67(8), 603-13.

Schacter, D. L., Addis, D. R., \& Buckner, R. L. (2008). Episodic Simulation of Future Events: Concepts, Data, and Applications. Ann.N.Y.Acad.Sci., 1124, 39-60.

Sescousse, G., Caldu, X., Segura, B., \& Dreher, J. C. (2013). Processing of primary and secondary rewards: a quantitative meta-analysis and review of human functional neuroimaging studies. Neurosci Biobehav Rev, 37(4), 681-96.

Singer, J. A., Blagov, P., Berry, M., \& Oost, K. M. (2013). Self-defining memories, scripts, and the life story: narrative identity in personality and psychotherapy. Journal of Personality, 81(6), 569582.

Singer, J. A., Rexhaj, B., \& Baddeley, J. (2007). Older, wiser, and happier? Comparing older adults' and college students' self-defining memories. Memory, 15(8), 886-98. 
Singer, J. L. (1998). Daydreams, the Stream of Consciousness, and Self-Representations. In R. F. Bornstein \& J. M. Masling (Eds.), Empirical Perspectives on the Psychoanalytic Unconscious (pp. 141-186). American Psychological Association.

Smallwood, J., Nind, L., \& O'Connor, R. C. (2009). When is your head at? An exploration of the factors associated with the temporal focus of the wandering mind. Conscious Cogn, 18(1), 118-25. Smallwood, J., Ruby, F. J. M., \& Singer, T. (2013). Letting go of the present: mind-wandering is associated with reduced delay discounting. Consciousness and Cognition, 22(1), 1-7.

Smallwood, J., \& Schooler, J. W. (2006). The restless mind. Psychol Bull, 132(6), 946-958.

Smallwood, J., \& Schooler, J. W. (2015). The Science of Mind Wandering: Empirically Navigating the Stream of Consciousness. Annual Review of Psychology, 66(1), 487-518.

Smallwood, J., Schooler, J. W., Turk, D. J., Cunningham, S. J., Burns, P., \& Macrae, C. N. (2011). Selfreflection and the temporal focus of the wandering mind. Consciousness and Cognition, 20(4), 1120-1126.

Song, X., \& Wang, X. (2012). Mind Wandering in Chinese Daily Lives - An Experience Sampling Study. PLOS ONE, 7(9), e44423.

Spreng, R. N., Mar, R. A., \& Kim, A. S. (2009). The common neural basis of autobiographical memory, prospection, navigation, theory of mind, and the default mode: a quantitative meta-analysis. J Cogn Neurosci, 21(3), 489-510.

Spreng, R. N., Stevens, W. D., Chamberlain, J. P., Gilmore, A. W., \& Schacter, D. L. (2010). Default network activity, coupled with the frontoparietal control network, supports goal-directed cognition. Neuroimage, 53(1), 303-17.

Stawarczyk, D., Cassol, H., \& D’Argembeau, A. (2013). Phenomenology of future-oriented mindwandering episodes. Front Psychol, 4, 425.

Stawarczyk, D., \& D’Argembeau, A. (2015). Neural correlates of personal goal processing during episodic future thinking and mind-wandering: An ALE meta-analysis. Human Brain Mapping, 36(8), 2928-2947. 
Stawarczyk, D., Majerus, S., Maj, M., Van der Linden, M., \& D’Argembeau, A. (2011). Mindwandering: Phenomenology and function as assessed with a novel experience sampling method. Acta Psychologica, 136, 370-381.

Stawarczyk, D., Majerus, S., Maquet, P., \& D’Argembeau, A. (2011). Neural correlates of ongoing conscious experience: both task-unrelatedness and stimulus-independence are related to default network activity. PLOS ONE, 6(2), e16997.

Sui, J., \& Humphreys, G. W. (2015). The Integrative Self: How Self-Reference Integrates Perception and Memory. Trends in Cognitive Sciences, 19(12), 719-728.

Svoboda, E., McKinnon, M. C., \& Levine, B. (2006). The functional neuroanatomy of autobiographical memory: a meta-analysis. Neuropsychologia, 44(12), 2189-2208.

Szpunar, K. K. (2010). Episodic future thought: An emerging concept. Perspectives on Psychological Science, 5, 142-162.

Szpunar, K. K., Watson, J. M., \& McDermott, K. B. (2007). Neural substrates of envisioning the future. Proc.Natl.Acad.Sci.U.S.A, 104(2), 642-647.

van der Meer, L., Costafreda, S., Aleman, A., \& David, A. S. (2010). Self-reflection and the brain: A theoretical review and meta-analysis of neuroimaging studies with implications for schizophrenia. Neurosci Biobehav Rev, 34(6), 935-946.

van Kesteren, M. T. R., Ruiter, D. J., Fernández, G., \& Henson, R. N. (2012). How schema and novelty augment memory formation. Trends in Neurosciences, 35(4), 211-219.

Whitfield-Gabrieli, S., Moran, J. M., Nieto-Castanon, A., Triantafyllou, C., Saxe, R., \& Gabrieli, J. D. (2011). Associations and dissociations between default and self-reference networks in the human brain. Neuroimage, 55(1), 225-32.

Zysset, S., Huber, O., Ferstl, E., \& von Cramon, D. Y. (2002). The anterior frontomedian cortex and evaluative judgment: an fMRI study. Neuroimage, 15(4), 983-91. 